\title{
Tracking dynamic interactions between structural and functional connectivity: a
}

\section{TMS/EEG-dMRI study}

Enrico Amico ${ }^{1,2,+}$, Olivier Bodart ${ }^{1}$, Mario Rosanova ${ }^{3}$, Olivia Gosseries ${ }^{1,5}$, Lizette Heine ${ }^{1}$, Pieter Van Mierlo ${ }^{4}$, Charlotte Martial ${ }^{1}$, Marcello Massimini ${ }^{3}$, Daniele Marinazzo ${ }^{2, *}$ and Steven Laureys ${ }^{1, *}$

1 Coma Science Group, Cyclotron Research Center \& GIGA Research Center, University and University Hospital of Liège, Liège, Belgium

2 Department of Data-analysis, University of Ghent, B9000 Ghent, Belgium

3 Department of Biomedical and Clinical Sciences "Luigi Sacco", University of Milan, Milan, Italy 4 Medical Image and Signal Processing Group, Department of Electronics and Information Systems, Ghent University-IBBT, B9000 Ghent, Belgium

5 Department of Psychiatry, University of Wisconsin, Madison, WI, USA

*authors contributed equally

+ corresponding author. E-mail: eamico@ulg.ac.be Tel: +32 43662362 Fax: +32 43662946

\section{Introduction}

Transcranial magnetic stimulation (TMS) has been used for more than 20 years to investigate connectivity and plasticity in the human cortex. By combining TMS with high-density electroencephalography (hd-EEG), one can stimulate any cortical area and measure the effects produced by this perturbation in the rest of the cerebral cortex (IImoniemi et al., 1997). It has been shown that cortical potentials elicited by TMS stimulation (TMS-evoked potentials, i.e., TEPs) mainly generate a significant EEG response during the first $300 \mathrm{~ms}$ in wakefulness (Massimini et al., 2005; Rosanova et al., 2009). The effects of TMS might also last for up to 600 ms, during their spread from the area of stimulation to remote interconnected brain areas (Bonato et al., 2006; Lioumis et al., 2009). To date, TMS/EEG recordings have provided new and reliable insights on the whole brain cortical excitability with reasonable spatial and excellent temporal resolution (Gosseries et al., 2015; Rogasch and Fitzgerald, 2013).

The amount of information contained in the hd-EEG response to TMS appears to contain inner signatures of the functional organization in a brain network. Two recent studies (Ferrarelli et al., 
2012; Rosanova et al., 2009) in healthy awake subjects showed that TMS can also induce EEG oscillations at different frequencies. The TMS pulse gives rise to different connected cortical regions in the brain, generating a complex EEG pattern composed of strong fluctuations at the "natural" frequency of the stimulated area. These oscillations are thought to reflect neurophysiological activity that is transiently elicited by the TMS pulse and possibly engaged through brain connections (Cona et al., 2011; Ferrarelli et al., 2012; Rosanova et al., 2009). Recently, researchers have started to investigate how the TMS/hd-EEG perturbation might be constrained and shaped by brain structure, either by exploring the correlation between TMS-induced interhemispheric signal propagation and neuroanatomy (Groppa et al., 2013; Voineskos et al., 2010), or by improving the modeling of the TMS-induced electric field using realistic neural geometry (Bortoletto et al., 2015; De Geeter et al., 2015). Besides, it has lately been shown that cortical networks derived from source EEG connectivity partially reflect both direct and indirect underlying white matter connectivity in a broad range of frequencies (Chu et al., 2015).

In this respect, the development of diffusion magnetic resonance imaging (dMRI) might add information on the structural architecture of the brain (Catani et al., 2002). The application of deterministic and probabilistic tractography methods allows for the spatial topography of the white matter, which represents bundles of coherently organized and myelinated axons (Song et al., 2002). The output of tractography algorithms permits anatomically plausible visualization of white matter pathways and has led to reliable quantification of structural connections between brain regions (i.e., the human connectome (Bullmore and Sporns, 2009; Sporns et al., 2005)). The purpose of this proof-of-concept paper is to investigate EEG changes of directed functional connectivity in the brain induced by TMS from both a functional and structural perspective, using multimodal modeling of source reconstructed TMS/hd-EEG recordings and dMRI 
tractography. The study of functional connectivity changes after the perturbation can possibly help in understanding the structure-function modulation caused by TMS (i.e., the extent to which TMS-induced EEG dynamics is constrained by white matter pathways) and the specific frequency bands of the involved brain regions. Taking the aforementioned recent findings as a starting point, we here aim to assess: 1) the role of the "natural frequencies" in the TMS-induced functional connectivity changes (Ferrarelli et al., 2012; Rosanova et al., 2009); 2) the extent to which functional connectivity, as a consequence of the induced perturbation, is shaped by brain structure.

We will first present the processing pipelines for TMS-EEG and dMRI data. Second, the mathematical methodology for the evaluation of the directed functional connectivity between brain regions and its correlation with the structural connectome will be presented. Finally, results obtained in a cohort of healthy volunteers will be presented and discussed.

\section{Materials and Methods}

\section{TMS/hd-EEG recordings: acquisition and preprocessing}

TMS/hd-EEG data were acquired in 14 healthy awake adults (6 males, age range 23-37 years) as published elsewhere (Casali et al., 2010; Casali et al., 2013; Rosanova et al., 2012). In brief, subjects were sitting on a reclined chair with eyes open looking at a fixation point on a screen. All participants gave written informed consent and underwent clinical examinations to rule out any potential adverse effect of TMS.

The TMS/hd-EEG experimental procedure, approved by the Local Ethical Committee of the University of Liège, was performed using a figure-of-eight coil driven by a mobile unit (eXimia 
TMS Stimulator, Nexstim Ltd., Finland), targeting two cortical areas (left superior parietal and left premotor) for at least 200 trials. These areas were selected for the following reasons: (i) they are easily accessible and far from major head or facial muscles whose activation may affect EEG recordings, (ii) previous TMS/EEG studies have been successfully performed in these areas during wakefulness (Casarotto et al., 2010; Massimini et al., 2005; Rosanova et al., 2009) and iii) because these areas are part of the fronto-parietal network, and thus are structurally and functionally highly connected. The left superior parietal and left premotor targets were identified on the subjects $3 \mathrm{D}$ T1 brain scan and reached through the neuronavigation system (NBS, Nexstim Ltd, Finland) using stereoscopic infrared tracking camera and reflective sensors on the subject's head and the stimulating coil.

Electrical brain activity was recorded using a 60-channel TMS-compatible EEG amplifier (Nexstim eXimia, Nexstim Plc, Finland) with a sample-and-hold circuit, which prevents the amplifier from saturation (see Supplementary Information for details).

Channels dominated by artifacts, such as $50 \mathrm{~Hz}$ noise, electrode movements, or strong TMS induced artifact were removed (maximum of 10 per session) and later interpolated. The session was discarded if the removed channels were clustered in such a way that interpolation would have been impossible or limited in accuracy. A minimum of 200 average trials was kept for further analyses. The percentage of excluded trials varied from subject to subject but was less than $10 \%$. TMS trials containing noise, muscle activity, or eye movements were detected and rejected (Rosanova et al., 2012), and the session was performed until 200 accepted trials were recorded, or a maximum of 400 per site (never reached in this healthy subjects population). ICA was also used as the last step in the signal preprocessing in some subjects, in order to remove artifacts from ocular movements or remaining $50 \mathrm{~Hz}$ noise, that could not be dealt with in the previous steps. 
Out of the initial 14 subjects, we excluded 5 of them for the superior parietal target and 2 for the premotor target, because of a low signal-to-noise ratio of TMS/EEG-evoked responses (i.e., when the ratio between post-stimulus to pre-stimulus amplitude is below 1.4, as in (Casali et al., 2010; Casali et al., 2013)). EEG data were referenced by means of the reference electrode standardization technique (Liu et al., 2015; Yao, 2001), downsampled at half of the original sampling rate (from $725 \mathrm{~Hz}$ to $362 \mathrm{~Hz}$ ), and bandpass filtered (2 to $80 \mathrm{~Hz}$ ).

Source reconstruction was performed as in (Casali et al., 2010; Casali et al., 2013). Conductive head volume was modeled according to the 3-spheres BERG method (Berg and Scherg, 1994) and constrained to the cerebral cortex that was modeled as a three-dimensional grid of 3004 fixed dipoles oriented normally to cortical surface. This model was adapted to the anatomy of each subject using the Statistical Parametric Mapping software package (SPM8, freely available at: http://www.fil.ion.bpmf.ac.uk/spm) as follows: binary masks of skull and scalp obtained from individual MRIs were warped to the corresponding canonical meshes of the Montreal Neurological Institute (MNI) atlas. Then, the inverse transformation was applied to the $\mathrm{MNI}$ canonical mesh of the cortex for approximating to real anatomy. Finally, the single trial distribution of electrical sources in the brain was estimated by applying the empirical Bayesian approach as described in (Mattout et al., 2006; Phillips et al., 2005).

In order to summarize significant functional measures over anatomically and/or functionally identifiable brain regions, the time courses of the 3004 reconstructed sources were then averaged into the specific 90 cortical and subcortical areas of the Automated Anatomical Labeling (AAL) (Tzourio-Mazoyer et al., 2002) parcellation (Fig.1), according to their position on the cortical mesh (Casali et al., 2010). Specifically, the AAL template was first registered onto the cortical mesh, allowing for a mapping between the 3004 fixed dipoles and the regions of interest (ROIs) of the template. Then, the time series of the dipoles falling in the same AAL brain 
region were averaged together.
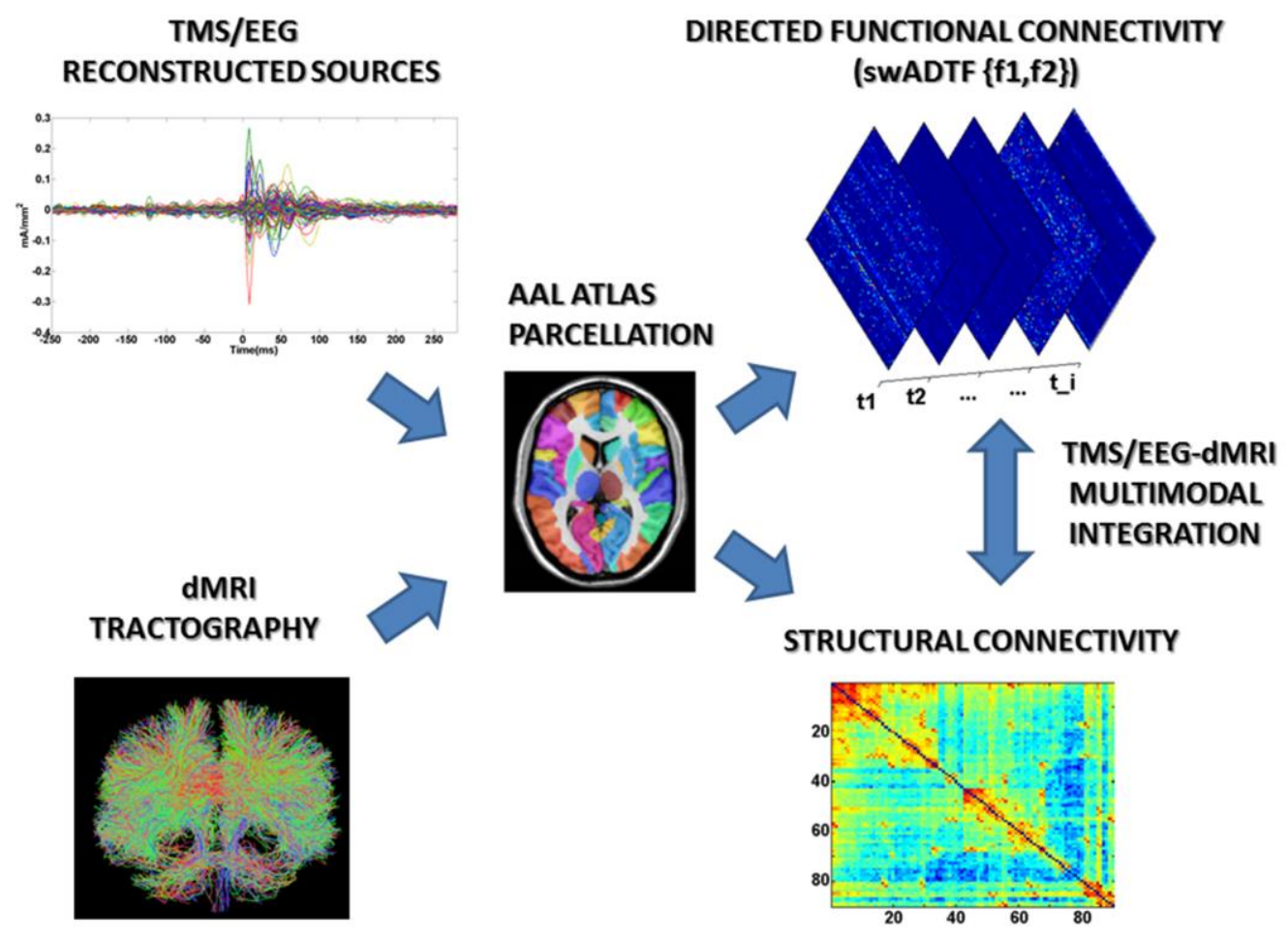

Figure 1: Flow chart of TMS/EEG-dMRI modeling. Up: the time courses of the 3004 reconstructed dipoles were averaged into the parcels of the Automated Anatomical Labeling (AAL) atlas (Tzourio-Mazoyer et al., 2002), consisting of 90 unique brain regions (cerebellar regions were excluded from the analysis). The 90 time courses obtained were modeled using spectrum-weighted adaptive directed transfer function (swADTF) (Van Mierlo et al., 2013; Van Mierlo et al., 2011). swADTF returns the causal interactions between the cortical regions ( $90 \times 90$ time varying directed functional connectivity matrices) at a specific frequency interval $(\mathrm{f} 1, \mathrm{f} 2)$. Bottom: for each dMRI dataset whole-brain probabilistic tractography was performed using a combination of FSL and MRTRIX (see Materials and methods). The AAL atlas was then used to segment the streamlines fiber bundles between each pair of ROls. Next, we determined the percentage of tracts between each pair of regions of the AAL template, resulting in a $90 \times 90$ structural connectivity matrix.

\section{dMRI data: acquisition and preprocessing}

A series of diffusion-weighted magnetic resonance images (dwi) of brain anatomy were 
acquired in each participant using a Siemens Trio Magnetom 3 Tesla system (Siemens Trio, University Hospital of Liege, Belgium). Diffusion-weighted images were acquired at a b-value of $1000 \mathrm{~s} / \mathrm{mm}^{2}$ using 64 encoding gradients that were uniformly distributed in space by an electrostatic repulsion approach (Jones et al., 1999). Voxels had dimensions of $1.8 \times 1.8 \times 3.3$ $\mathrm{mm}^{3}$ and volumes were acquired in 45 transverse slices using a $128 \times 128$ voxel matrix. A single T1-weighted 3D magnetization-prepared rapid gradient echo sequence (MPRAGE) image, with isotropic resolution of $1 \mathrm{~mm}^{3}$, was also acquired for each subject.

Diffusion volumes were analysed using typical preprocessing steps in dMRI (Caeyenberghs et al., 2012; Zalesky et al., 2014). Eddy current correction for each participant was achieved using FDT, v2.0, the diffusion toolkit within FSL 5.0 (FMRIB Software Library; http://www.fmrib.ox.ac.uk/fsl). Rotations applied to the diffusion-weighted volumes were also applied to the corresponding gradient directions (Leemans and Jones, 2009). A fractional anisotropy (FA) image was estimated using weighted linear least squares fitted to the log-transformed data for each subject.

\section{dMRI data: registration of the anatomical image and atlas parcellation}

We segmented each subject's T1-weighted image into whole-brain white matter (WM), gray matter (GM), and cerebrospinal fluid (CSF) masks using FAST, part of FSL (FMRIB Software Library $v$ 5.0). The corresponding white matter mask image was registered without resampling to the relevant dwi series (Smith et al., 2004). Next, the AAL atlas was first registered to the T1 space using linear (FSL flirt) and non-linear warping (FSL FNIRT) in order to achieve the best registration into each subject's space. Then, the single subject AAL template was finally registered without resampling to the dwi space using the affine transform resulting from the WM 
registration. This transformation matrix was also applied to the T1-derived GM mask which was used as termination mask for the tractography analysis.

\section{dMRI data: tractography and connectome construction}

The fiber response model was estimated for each subject from the high $b$-value $(b=1000 \mathrm{~s} /$ $\mathrm{mm}^{2}$ ) diffusion-weighted images. A mask of single fiber voxels was extracted from the thresholded and eroded FA images (Tournier et al., 2004; Tournier et al., 2008). Using non-negativity constrained spherical deconvolution, fiber orientation distribution (FOD) functions were obtained at each voxel using the MRTRIX3 package (J-D Tournier, Brain Research Institute, Melbourne, Australia, https://github.com/jdtournier/mrtrix3) (Tournier et al., 2012). For both the response estimation and spherical deconvolution steps we chose a maximum harmonic order $l_{\max }$ of 6 , based on a successful application of constrained spherical deconvolution with similar b-value and number of directions (Roine et al., 2015).

Probabilistic tractography was performed using randomly placed seeds within subject-specific white matter masks, registered as mentioned in the latter. Streamline tracking settings were as follows: number of tracks $=10$ million, FOD magnitude cutoff for terminating tracks $=0.1$, minimum track length $=5 \mathrm{~mm}$, maximum track length $=200 \mathrm{~mm}$, minimum radius of curvature $=$ $1 \mathrm{~mm}$, tracking algorithm step size $=0.5 \mathrm{~mm}$. Streamlines were terminated when they extended out of the WM-GM mask interface, or could not progress along a direction with an FOD magnitude or curvature radius higher than the minimum cutoffs.

The streamlines obtained were mapped to the relevant nodes defined by the AAL parcellation registered in the subject's dwi space, using MRTRIX3 (Tournier et al., 2012). Each streamline termination was assigned to the nearest gray matter parcel within a $2 \mathrm{~mm}$ search radius. The resulting connectome was finally examined by determining the connection density (number of 
streamlines per unit surface) between any two regions of the AAL template, as in (Caeyenberghs et al., 2012) (see also Fig.1). This correction was needed to account for the variable size of the cortical ROls of the AAL template (Hagmann et al., 2008).

\section{TMS/hd-EEG directed functional connectivity estimation: spectrum-weighted adaptive} directed transfer function

We evaluated directed functional connectivity between the 90 EEG reconstructed brain signals (see section TMS/hd-EEG recordings: acquisition and preprocessing) by using an extension of a data-driven technique based on Granger causality (Granger, 1969). According to Granger causality, if a signal $x_{1}$ "Granger causes" (or "G-causes") a signal $x_{2}$, then past values of $x_{1}$ should contain information that helps to predict $x_{2}$ above and beyond the information contained in past values of $x_{2}$ alone (Granger, 1969). This statistical concept can be mathematically modeled as a multivariate autoregressive model (MVAR):

$$
x_{n}=\sum_{m=1}^{p} A_{m} x_{n-m}+e_{n}
$$

Where, for a number $K$ of signals, $x_{n}$ is the matrix containing the $K$ signals at time $n, e_{n}$ is a matrix containing the uncorrelated white noise at time $n, p$ is the model order (i.e., how many past values are taken into account) and $A_{m}$ is the $K \times K$ coefficient matrix for delay $m$.

The Directed Transfer Function (DTF) is a measure based on Granger causality to analyze the propagation of activity between multiple signals in the frequency domain, by means of a multivariate model of spectral coefficients (Kaminski and Blinowska, 1991): 


$$
D_{T F}(f)=\left|H_{i j}(f)\right|^{2}
$$

The information on the interactions between signals (i.e., brain regions, in our case) in the frequency domain is now contained in the $K \times K$ matrix $H$ (the equivalent of $A$ in eq. 1 in the frequency domain), also known as transfer matrix of the model, which contains information on the directed interactions between signal $x_{i}$ and $x_{j}$ at frequency $f$.

These models assume stationary signals as input. In order to cope with the non-stationary nature of most natural signals, an extension of the DTF, the adaptive directed transfer function (ADTF) (Arnold et al., 1998; Astolfi et al., 2008; Wilke et al., 2008) was proposed. To adapt the autoregressive model to non-stationary signals it was allowed to the coefficients of the autoregressive model (i.e., matrix $\mathrm{H}$ in eq. 2) to vary in time, by means of a Kalman filtering procedure (Arnold et al., 1998) (the interested reader may refer to (Van Mierlo et al., 2013; Van Mierlo et al., 2011) for details on the methodology).

This time-variant multivariate autoregressive (TVAR) model with time varying parameters has been successfully used for connectivity modeling of epileptic intracranial EEG data (Van Mierlo et al., 2013; Van Mierlo et al., 2011). In order to take into account the non-stationary nature of the TMS pulse in our EEG source-reconstructed signals, we here adopted the spectrum-weighted version of the adaptive directed transfer function (swADTF)(Van Mierlo et al., 2013; Van Mierlo et al., 2011):

$$
\operatorname{swADTF}_{i j}(t)=\frac{\sum_{f=f_{1}}^{f_{2}}\left|H_{i j}(f, t)\right|^{2} \sum_{k=1}^{K}\left|H_{j k}(f, t)\right|^{2}}{\sum_{k=1}^{K} \sum_{f=f_{1}}^{f_{2}}\left|H_{i k}(f, t)\right|^{2} \sum_{k=1}^{K}\left|H_{j k}(f, t)\right|^{2}}
$$


where $H_{i j}(f, t)$ in eq. 3 is the time-variant transfer matrix of the system describing the directed functional connectivity from signal $j$ to $i$ at frequency $f$ at time $t$, for each of the $K$ signals.

Each term $H_{i j}(f, t)$ is weighted by the autospectrum of the sending (in this case j) signal.

The swADTF allows us to investigate directional interactions between all the signals at a predefined frequency band over time. The measure weights all outgoing directed functional connectivity present in the terms $H_{i j}(f, t)$ by the power spectrum of the sending signal $\mathrm{j}$, and therefore does not depend on the power amplitude. Each swADTF value corresponds to the directed time-variant strength of the directed functional connectivity between two nodes. This dynamic interaction between nodes can also be represented as a series of time-varying directed matrices (see also Fig.1). The swADTF is normalized so that the sum of incoming directed functional connectivity into a channel at each time point is equal to 1 :

$$
\sum_{k=1}^{K} s w A D T F_{i k}(t)=1
$$

\section{TMS/EEG-dMRI multimodal integration: outdegree computation and statistical} assessment

We computed directed functional connectivity (swADTF) on the brain network defined by the anatomical atlas (AAL) reconstructed sources for each subject. A detailed discussion on the implementation and the setup of the parameters can be found in (Van Mierlo et al., 2011). 
The swADTF was calculated in 3 frequency bands: $\alpha \quad(8-12 \mathrm{~Hz}), \beta(13-20 \mathrm{~Hz}), \quad \beta 2 / \gamma \quad(21-50$ $\mathrm{Hz}$ ), as in (Rosanova et al., 2009). We chose these three bands for reasons of comparison with the study by Rosanova et al., which provided evidence that TMS on healthy awake subjects consistently evoked dominant EEG oscillations in different cortical areas.

In order to track modulations of directed functional connectivity due to TMS, we considered 3 different non-overlapping windows of 300 ms: a "baseline", pre-stimulus, extended from -300 ms to $0 \mathrm{~ms}$ before the TMS pulse; a "post-stimulus", after TMS pulse, which captures the dynamics from 20 to $320 \mathrm{~ms}$ after the pulse (the first $20 \mathrm{~ms}$ were discarded to minimize the effect of possible artifacts occurring at the time of stimulation, (Rogasch et al., 2013; Rosanova et al., 2009)); a "late post-stimulus", from 320 to 620 ms after the stimulation.

We obtained the mean global outgoing flow from a region $j$ before and after the stimulation by averaging the swADTF time courses in each of the three time windows and by summing the average amount of directed connectivity transferred from $j$ to each node of the network. In network terms, this quantity is called Outdegree. In our case, for each frequency band and window (i.e., baseline or post-stimulus):

$$
\text { Outdegree }_{j}=\sum_{k=1}^{K} C_{j k}, \quad \forall k, j=1 \ldots K
$$

where $K=90$ in our case (i.e., the number of AAL regions), and $C$ is the connectivity matrix constructed by averaging the swADTF time courses within each window. All self-edges were set to 0 . By using this procedure we aimed to obtain an illustrative snapshot of the total directed 
functional connectivity from a region $j$ at a specific stage of the TMS process (i.e., baseline or post-stimulus).

In order to detect significant group changes in the Outdegree before and after the stimulation, a two-sample t-test of the post-stimulus Outdegree against the correspondent baseline Outdegree was performed in each region. Post stimulus Outdegree values were considered significant at $p<0.05$, False Discovery Rate (FDR) corrected for multiple comparisons over the 90 brain regions, the 3 frequency bands and the 2 sites of stimulation.

Furthermore, in order to investigate how the integration and segregation of the functional brain network change after the TMS pulse, we evaluated some key topological properties of the directed functional connectivity networks, before and after TMS-pulse. The following network measures were computed: clustering coefficient, betweenness centrality, rich club coefficient, global efficiency (Bullmore and Sporns, 2009; Sporns, 2011), using the Brain Connectivity Toolbox ((brain-connectivity-toolbox.net, (Rubinov and Sporns, 2010)). The differences pre-post TMS pulse of these network measures were then tested based on Wilcoxon signed-rank test, and then FDR corrected for multiple comparisons over the 90 brain regions, the 3 frequency bands and the 2 sites of stimulation.

Finally, for each subject, the structural degree of a node $j$ (SCdegree) was simply calculated from the structural connectivity matrix $S$ by summing over its columns.

$$
\text { SCdegree }_{j}=\sum_{k=1}^{K} S_{j k}, \quad \forall j=1 \ldots K,
$$




\section{Structure-function correlations and statistical assessment}

The dynamic interaction between regions modeled by SwADTF can be represented as a series of time-varying directed connectivity matrices (see also Fig.1). In each frequency band, dynamic spatial correlation was defined as the mean row-by-row Pearson's correlation at each time point between each subject's directed functional connectivity matrix and the correspondent structural connectivity matrix. The $95 \%$ confidence intervals for the Pearson's correlation distribution at the baseline were calculated by using a non-parametric bootstrap procedure (Efron and Tibshirani, 1986). Specifically, the correlation coefficient was recomputed $\mathrm{n}=100$ times on the resampled data obtained by $\mathrm{n}$ random permutations of the values in the directed functional connectivity matrices at each time point of the baseline, while leaving the structural connectivity matrix unchanged. The empirical distribution of the resampled dynamic spatial correlation values at the baseline was used to approximate the sampling distribution of the statistic. A 95\% confidence interval for the baseline was then defined as the interval spanning from the 2,5th to the 97,5 th percentile of the obtained distribution. Values in dynamic spatial correlation that were falling outside this interval were considered significantly different from the baseline correlation.

\section{Results}

The significant differences (Table 1) in directed functional connectivity, between baseline and post-stimulus window, across cortical regions after TMS perturbation are illustrated by projecting the OutDegree onto the anatomical template (Fig. 2). In the first $300 \mathrm{~ms}$ after the 
pulse, the two sites of stimulation have significant Outdegree peaks at different frequency bands. However, we did not find any significant differences between Outdegree values of baseline and late post-stimulus windows.

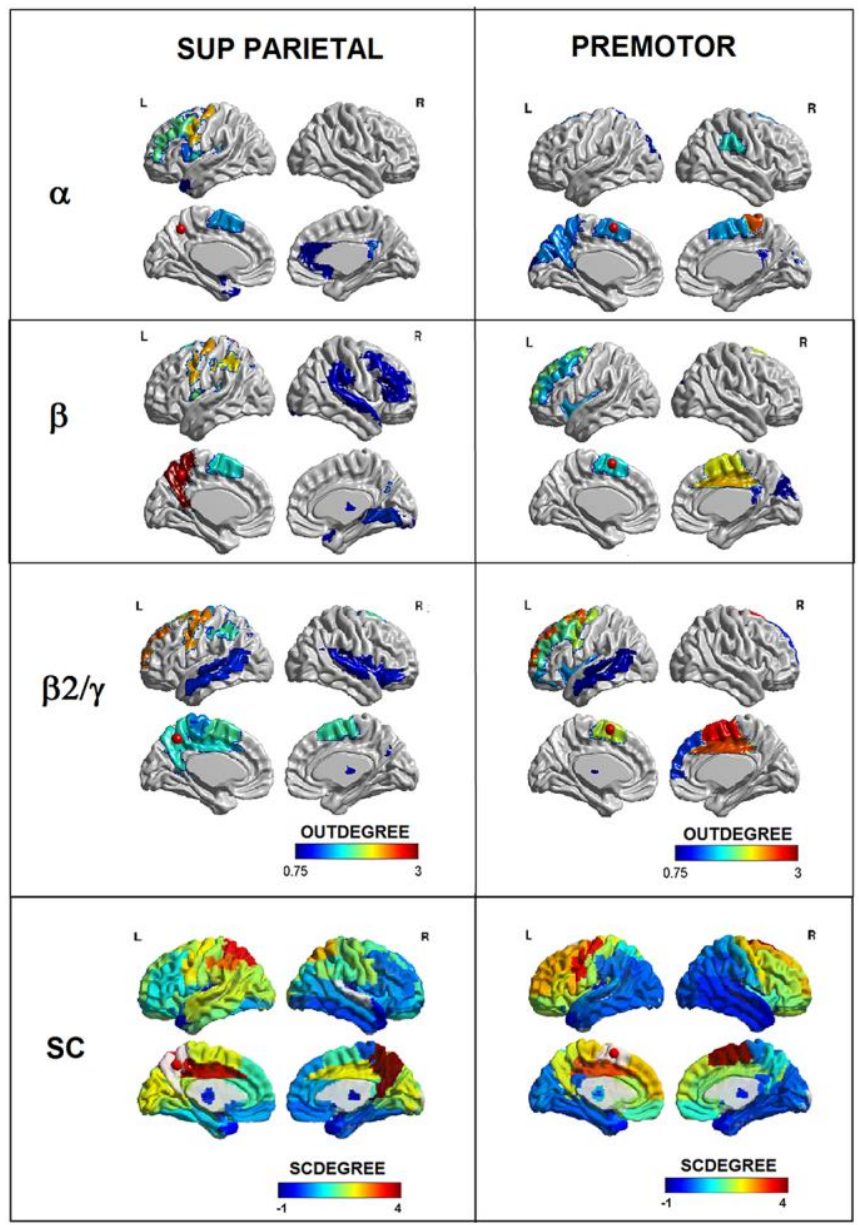

Figure 2: Directed functional connectivity across cortical regions after TMS. First three rows : snapshot of differences between baseline and post TMS stimulus directed functional connectivity (i.e., Outdegree) at $p<0.05$, FDR corrected (see Materials and Methods) across cortical regions, for the three predefined frequency bands ( $\alpha, \beta, \beta 2 / \gamma$ (Rosanova et al., 2009)), obtained by averaging the swADTF time courses from 20 to $320 \mathrm{~ms}$ after the pulse. The red circles coarsely indicate the target areas of stimulation . Bottom row: z-scored map of the structural connectivity profile of the two stimulated regions, representing amount of connecting the superior parietal cortex (left) and the premotor cortex (right). The red circles coarsely indicate the target areas of stimulation. Note that the superior parietal cortex has a maximum of directed functional connectivity in the $\beta$ 
band in proximity of the stimulation site, whereas the premotor cortex has a maxima in the $\beta 2 / \gamma$ band, more spread towards the hemisphere controlateral to the stimulation site. These brain images were obtained using BrainNet Viewer (Xia et al., 2013).

TMS SITE:

LEFT SUPERIOR PARIETAL

\section{TMS SITE:}

LEFT PREMOTOR

\begin{tabular}{|c|c|c|c|c|c|}
\hline AAL REGION & $\begin{array}{l}\text { INCREASE } \\
\text { (\%) }\end{array}$ & F.B. & AAL REGION & $\begin{array}{l}\text { INCREASE } \\
\text { (\%) }\end{array}$ & F.B. \\
\hline Precentral_L & 120 & $\alpha$ & Supp_Motor_Area_L & 210 & $\alpha$ \\
\hline Frontal_Mid_L & 133 & $\alpha$ & Supp_Motor_Area_R & 190 & $\alpha$ \\
\hline Frontal_Inf_Oper_L & 70 & $\alpha$ & Cingulum_Post_R & 60 & $\alpha$ \\
\hline Rolandic_Oper_L & 60 & $\alpha$ & Cuneus_L & 120 & $\alpha$ \\
\hline Supp_Motor_Area_L & 40 & $\alpha$ & Occipital_Sup_L & 90 & $\alpha$ \\
\hline \multirow[t]{3}{*}{ Cingulum_Post_R } & 53 & $\alpha$ & SupraMarginal_R & 50 & $\alpha$ \\
\hline & & & Sup_Parietal_L & 80 & $\alpha$ \\
\hline & & & Paracentral_Lobule_R & 110 & $\alpha$ \\
\hline Precentral_L & 150 & $\beta$ & Frontal_Sup_L & 185 & $\beta$ \\
\hline Frontal_Mid_R & 70 & $\beta$ & Frontal_Mid_L & 150 & $\beta$ \\
\hline Rolandic_Oper_L & 50 & $\beta$ & Supp_Motor_Area_L & 220 & $\beta$ \\
\hline Supp_Motor_Area_L & 90 & $\beta$ & Supp_Motor_Area_R & 260 & $\beta$ \\
\hline Parietal_Inf_L & 167 & $\beta$ & Insula_L & 50 & $\beta$ \\
\hline \multirow[t]{4}{*}{ Sup_Parietal_L } & 280 & $\beta$ & Cingulum_Mid_R & 52 & $\beta$ \\
\hline & & & Cingulum_Post_R & 35 & $\beta$ \\
\hline & & & Cuneus_R & 30 & $\beta$ \\
\hline & & & Cingulum_Mid_R & 45 & $\beta$ \\
\hline Precentral_L & 233 & $\beta 2 / \gamma$ & Precentral_L & 118 & $\beta 2 / \gamma$ \\
\hline Frontal_Sup_L & 152 & $\beta 2 / \gamma$ & Frontal_Sup_L & 363 & $\beta 2 / \gamma$ \\
\hline Supp_Motor_Area_L & 126 & $\beta 2 / \gamma$ & Frontal_Mid_L & 203 & $\beta 2 / \gamma$ \\
\hline Supp_Motor_Area_R & 130 & $\beta 2 / \gamma$ & Frontal_Mid_Orb_L & 153 & $\beta 2 / \gamma$ \\
\hline Cingulum_Mid_L & 100 & $\beta 2 / \gamma$ & Supp_Motor_Area_L & 330 & $\beta 2 / \gamma$ \\
\hline Parietal_Inf_L & 110 & $\beta 2 / \gamma$ & Supp_Motor_Area_R & 432 & $\beta 2 / \gamma$ \\
\hline \multirow[t]{5}{*}{ Sup_Parietal_L } & 150 & $\beta 2 / \gamma$ & Frontal_Sup_Medial_R & 65 & $\beta 2 / \gamma$ \\
\hline & & & Insula_L & 33 & $\beta 2 / \gamma$ \\
\hline & & & Cingulum_Mid_R & 110 & $\beta 2 / \gamma$ \\
\hline & & & Thalamus_L & 33 & $\beta 2 / \gamma$ \\
\hline & & & Temporal_Mid_L & 53 & $\beta 2 / \gamma$ \\
\hline
\end{tabular}


Table 1: Percentage increase before/after stimulation for the AAL areas illustrated in Fig. 2 where directed functional connectivity after TMS was significantly higher than baseline $(p<0.05$, FDR corrected, see Materials and Methods), for the two sites of stimulation (left superior parietal and left premotor) and the three different frequency band (F.B., i.e., $\alpha, \beta, \beta 2 / \gamma$ ). Top increment for each site of stimulation is highlighted in bold.

In particular, the superior parietal cortex has a maximum of directed functional connectivity in the $\beta$ band in proximity of the stimulation site, whereas the premotor has a maxima in the $\beta 2 / \gamma$ band, more spread towards the controlateral hemisphere. Statistical comparisons (i.e., double-sided t tests, $p<0.05$ ) of directed functional connectivity peaks on the two sites of stimulation using a finer frequency step (i.e., every $2 \mathrm{~Hz}$ ) provide further evidence that each target region has Outdegree maxima at different frequencies (i.e., within the $\beta$ range for the superior parietal cortex, within the $\beta 2 / \gamma$ for the premotor cortex, Fig. 3).
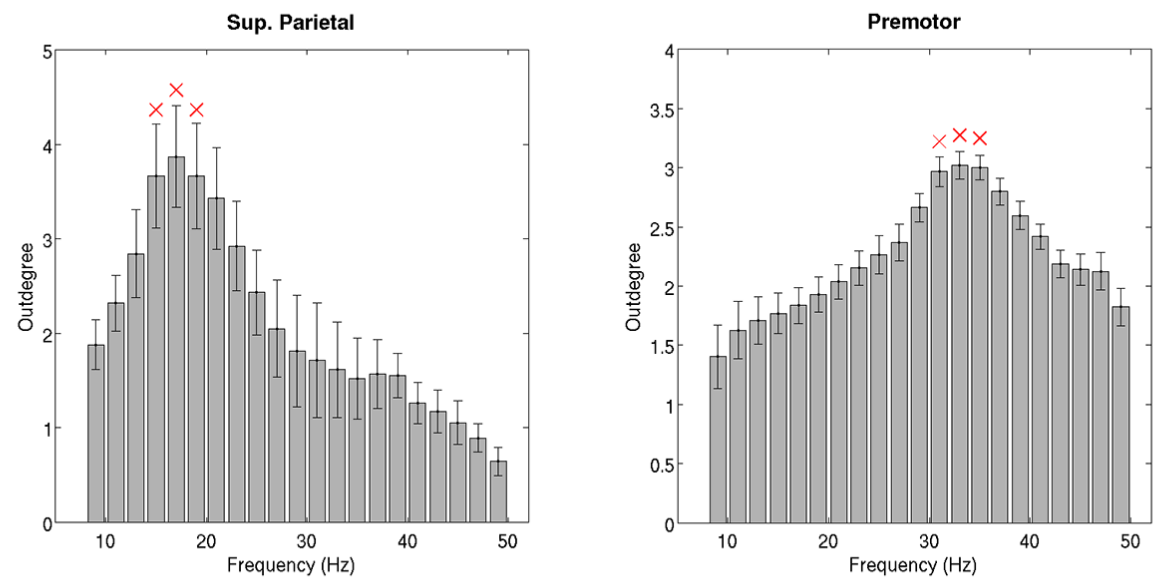

Figure 3: Peaks of directed functional connectivity for the two stimulated regions.. Barplots show Outdegree values for the two sites of stimulation (left, sup parietal; right, premotor) when using finer frequency ranges ( $2 \mathrm{~Hz}$ steps). Errorbars indicate standard error over subjects. The red crosses on the top of the bars indicate that the Outdegree value is significanlty different for the two target regions (double-sided t test, $p<0.05$ ). Note how the two sites of stimulation have Outdegree maxima in different frequency ranges (i.e., within the $\beta$ 
range for the superior parietal cortex, within the $\beta 2 / \gamma$ for the premotor cortex).

The peaks in directed functional connectivity at different frequencies in the areas depicted in Fig. 2 confirm the hypothesis that different brain regions might be normally tuned to oscillate at a characteristic rate (i.e., natural frequency) (Ferrarelli et al., 2012; Rosanova et al., 2009). Furthermore, despite the fast and chaotic functional response generated by the TMS pulse in the brain network, the cortical regions significantly recruited by TMS seem to maintain peaks of functional activation at the specific natural frequency consistently over time (Fig. 4, see also the movies in Supplementary Material). Notably, while the connectivity peaks of the premotor site follow the specific differences in the power spectrum, the peaks of directed functional connectivity in the superior parietal site appear to be more connectivity-specific (Fig. S1 in the supplementary information).
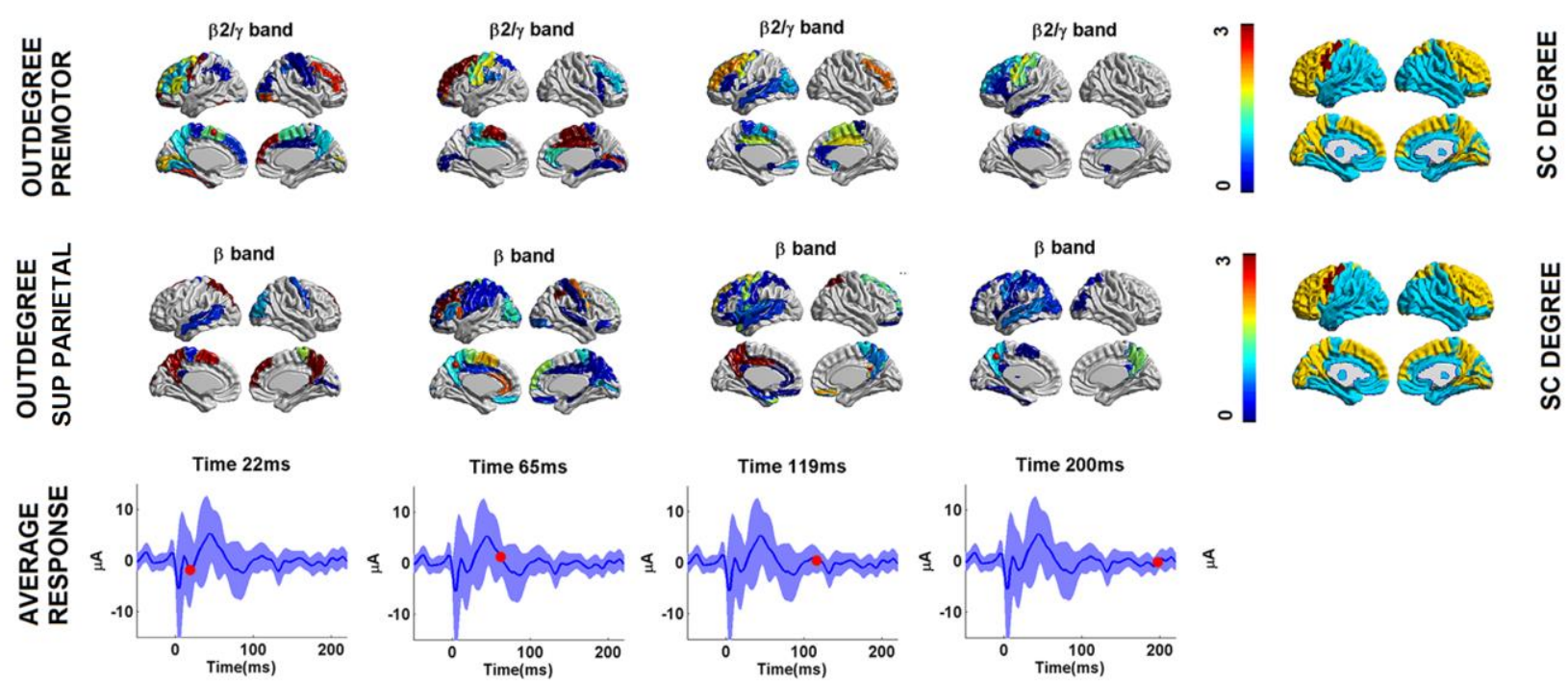

Figure 4: Flow of directed functional connectivity over time. Selected frames from the video in the Supplementary Material, of the functional connectivity across cortical regions, for both sites of stimulation (top two rows), after the TMS pulse. The temporal changes of the Outdegree for the natural frequency of the site of stimulation (i.e., $\beta$ for superior parietal, $\beta 2 / \gamma$ for premotor (Rosanova et al., 2009), see also Fig.2) is illustrated 
in four different time points after the TMS pulse. The red circles coarsely indicate the target areas of stimulation . A grand average plot of the EEG reconstructed sources is also shown on the third row. The red marker denotes the time instant along the ERP time series for the associated frame (on top). A z-scored map of the group averaged SCdegree (fixed, does not change over time) is depicted on the right. These brain images were obtained using BrainNet Viewer (Xia et al., 2013).

It is also worth mentioning that none of the network measures computed on the directed functional connectivity matrices before and after the TMS pulse survived to the statistical significance threshold ( $p<0.05$ FDR corrected, see Materials and Methods). This might be due to the small sample size of the cohort under study and to the within-subject variability of the response to TMS (see Limitations).

The dynamic spatial correlation between the directed functional connectivity (swADTF) and the connectome, for the two different sites of stimulation (i.e., left superior parietal and left premotor) and for each of the three chosen frequency bands (i.e., $\alpha, \beta, \beta 2 / \gamma$ ) deviates from baseline after the TMS pulse (Fig. 5). This global network behavior does not depend on the subject or the stimulation site. The stable baseline configuration is then recovered after 200-300 ms, depending on the frequency band. 

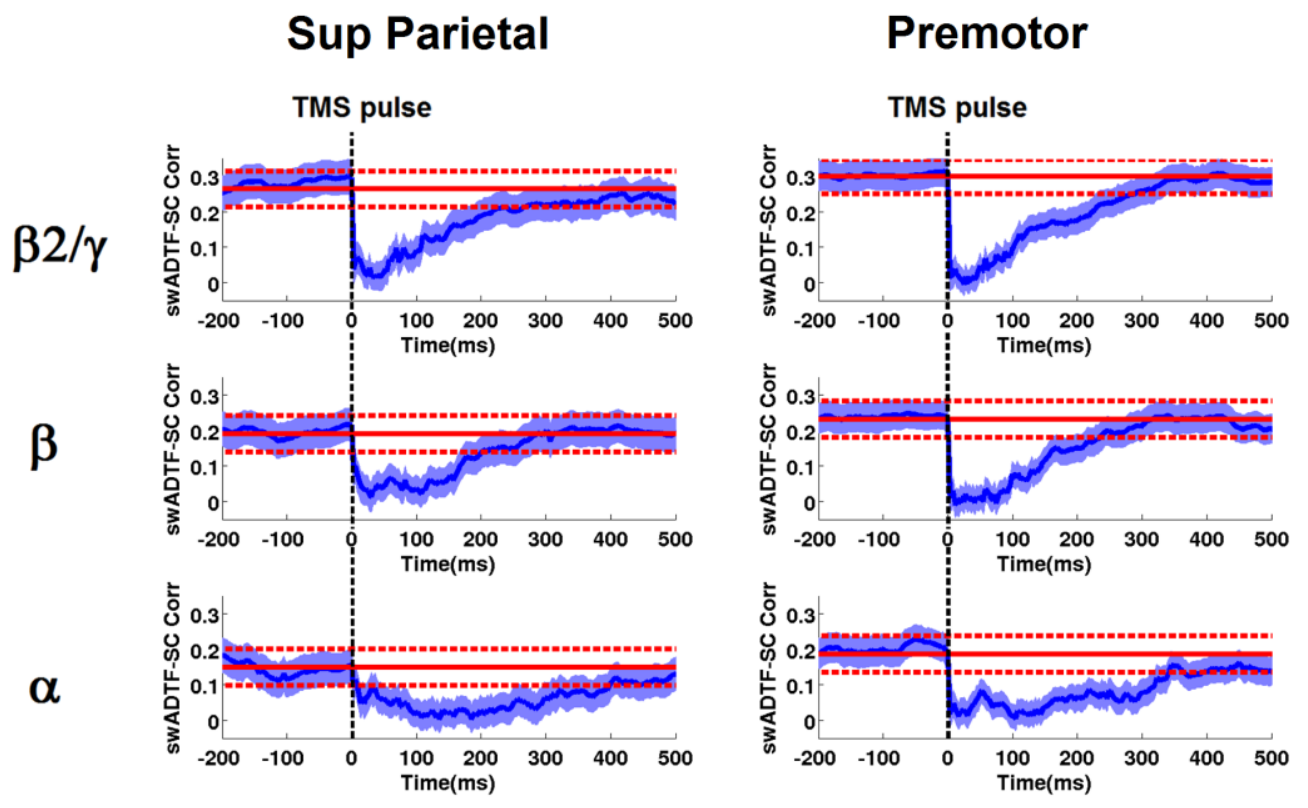

Figure 5: Time-varying spatial correlation between directed functional connectivity and structural connectivity. Each plot shows the average over subjects of the dynamic spatial correlation between the directed functional connectivity (swADTF) matrices and the structural connectivity (SC) in function of time (blue line, standard error in shaded blue), for the three different frequency bands ( $\alpha, \beta, \beta 2 / \gamma$, (Rosanova et al., 2009)). The red line indicates the mean baseline value, the dashed lines represent $95 \%$ confidence interval of the empirical baseline distribution (see Materials and Methods). Note the TMS-induced decrease in the observed structure-function correlation, for both stimulation sites and in each frequency band.

Specifically, this TMS-induced modulation of EEG rhythms over the brain network is more pronounced (i.e., higher deviation from the baseline correlation) and faster in the $\beta 2 / \gamma$ and $\beta$ bands, while the return to baseline is slower and less pronounced in the $\alpha$ band. The evidence that different brain area can be normally tuned by TMS to oscillate at a characteristic rate (i.e., natural frequency) might also explain the drop in structure-function correlation depicted in Fig. 5.

In fact, assuming that each of the 90 AAL cortical regions respond to TMS by oscillating at its 
peculiar natural frequency, the emergence of this complex between-band interaction might generate a consequent deflection in the within-band structure-function correlation (Fig. 5). This effect might be due to the region-specific variability in the intensity and the duration of the cortical response to TMS at the different natural frequencies, but it might also depend on the degree to which each recruited region is structurally connected to the rest of the network.

To further investigate whether the structural connectivity profiles of the stimulated regions influence subsequent TMS-evoked connectivity, we evaluated the local dynamic spatial correlation between the directed functional connectivity (swADTF) for the targeted cortical regions and the connectome, for both sites of stimulation (Fig. 6). Notably, the structure-function correlation significantly increases over time in the right premotor cortex after TMS, when its natural frequency band (i.e., $\beta 2 / \gamma$ ) was taken into consideration. This effect was not reproduced in the superior parietal area (Fig. 6). This increase in the structure-function correlation seems to be specific of the TMS-evoked response, and dependent on the cortical module elicited, as we did not observe such response when the same area was not stimulated, or in regions where the TMS pulse was most likely not causing any significant detectable effect (e.g., deep subcortical structures such as putamen, see also Supplementary Figure S2 and S3). 


\section{SUP PARIETAL}
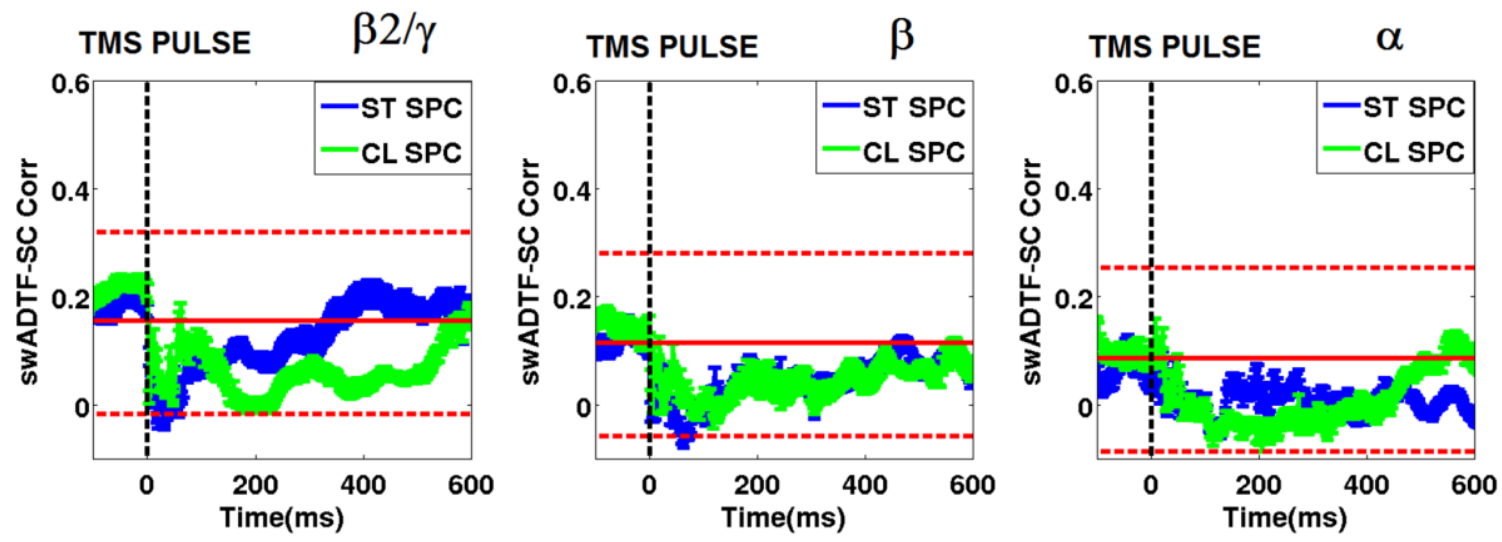

PREMOTOR
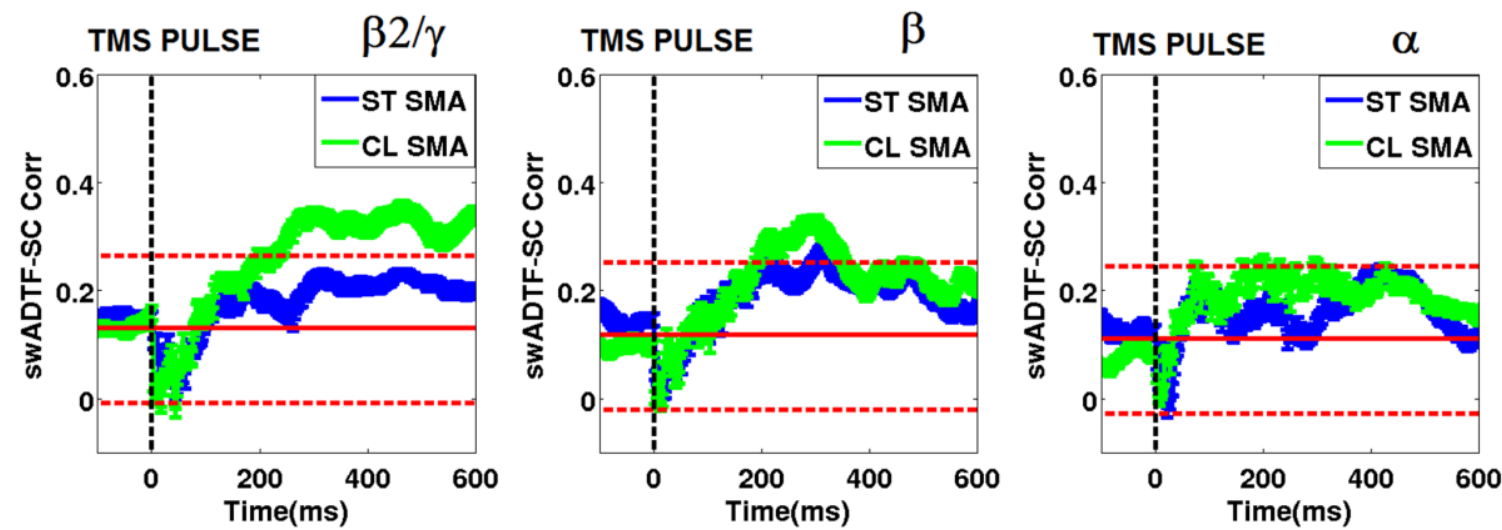

Figure 6: Time-varying spatial correlation for the stimulated cortical regions. Each row shows the average over subjects of the dynamic spatial correlation (blue and green line, standard error in shaded blue and green) between the directed functional connectivity (swADTF) and structural connectivity (SC) for the AAL ROIs comprising left and right premotor areas (stimulated and controlateral, i.e., ST SMA, CL SMA) and the left and right superior parietal (stimulated and controlateral, i.e., ST PCC, CL PCC) respectively, for the three different frequency bands (i.e., $\alpha, \beta, \beta 2 / \gamma$ respectively (Rosanova et al., 2009) ). The continuous red line indicates the mean baseline value, the dashed lines represent $95 \%$ confidence interval of the empirical baseline distribution (see Materials and Methods). Note the constant increase over time in the structure-function correlation for the controlateral SMA following the TMS pulse, when taking into account its natural frequency (i.e., $\beta 2 / \gamma$, Fig.2). 


\section{Discussion}

In this work we studied for the first time the interplay between directed functional connectivity computed from TMS reconstructed EEG sources and the connectome extracted from whole-brain dMRI tractography in a cohort of healthy volunteers. We aimed to assess: 1) whether natural frequencies of the stimulated areas play a role in the TMS-induced functional connectivity changes and 2) to what extent these functional connectivity changes are shaped by brain structure. Below follows a detailed discussion of the findings. First, this work confirms the hypothesis that different rhythms in the brain emerge after TMS, which was the first aim of our study. This dynamic interaction at different natural frequencies seems to reflect intrinsic properties of cortical regions, and the way those are interconnected (Cona et al., 2011; Rosanova et al., 2009). Previous studies revealed that distant areas, when activated by TMS, responded with oscillations closer to their own "natural" frequency (Ferrarelli et al., 2012; Rosanova et al., 2009). Our analysis on peaks of significant changes in directed functional connectivity at different frequency bands corroborated the hypothesis that TMS evokes dominant oscillation in different cortical areas at a characteristic rate. These findings are in line with previous studies (Ferrarelli et al., 2012; Rosanova et al., 2009), where the authors showed that TMS on healthy awake subjects consistently evokes EEG oscillations with dominant frequencies that depend on the site of stimulation. In particular, when stimulated, the superior parietal cortex was shown to respond to TMS in the $\beta$ band and the premotor cortex in $\beta 2 / \gamma$

(Rosanova et al., 2009). Here, we have tested and validated the natural frequency hypothesis by tracking directed functional connectivity interactions between brain regions, and by comparing their response before and after TMS.

Each stimulated area appeared to mainly respond to the stimulation by being functionally 23 
elicited in specific "natural" frequency bands, i.e., $\beta$ for superior parietal and $\beta 2 / \gamma$ for premotor (Fig. 2, Fig 3, Table 1). Furthermore, these peaks of functional changes at the natural frequency of the stimulation site after TMS seem to be quite stable over time (Fig.4, see also the movies in Supplementary Material). Interestingly, the premotor cortex also showed a less pronounced activation in the $\beta$ band (Fig. 2). This could arise from the recruitment after the stimulation of the primary motor cortex which is also known to display a dominant frequency in that frequency range (Van Der Werf and Paus, 2006).

Secondly, our analysis permitted to evaluate the dynamic interactions between directed functional connectivity and anatomical connectivity, before and after TMS, which was the second aim of our study.

We compared structural and directed functional connectivity at the whole network level for different EEG bands ( $\alpha, \beta, \beta 2 / \gamma$, Fig. 5). The interplay between directed functional connectivity and structural connectivity at baseline is in line with findings reported in recent fMRI-dMRI studies (Barttfeld et al., 2015), where the rich repertoire of brain states do not necessarily correlate with the structural pattern. Here, our directed functional connectivity approach also allowed the investigation of systematic TMS-induced perturbations of the system, extending the insight on the relationship between structure and function.

We observed a temporary decrease in the correlation between directed connectivity and structural connectivity after TMS. Assuming that each region in the brain reacts to the perturbation at a characteristic operating frequency, then the decrease in function-structure correlation in each frequency (Fig. 5) might be caused by the complex pattern of between-frequency interactions rising after TMS in the whole-brain network. The return to baseline might then depend on two things: one is the temporal duration of the functional 
activation of the elicited area; the second is the extent to which it is related to its structural connectivity pattern.

These considerations brought us to explore the link between the "natural" frequency response of the stimulated cortical areas and their structural architecture. Interestingly, for the premotor cortex controlateral to the stimulation site the correlation between directed functional connectivity at the natural frequency and structural connections increases after the stimulation and reveals a long-lasting effect over time (Fig.6). The fact that this effect is not reproduced for the superior parietal cortex might be due to a number of reasons. First, it has been shown that this area has lower cortical excitability than the premotor cortex, and thus it is more difficult to trigger (Ferrarelli et al., 2012; Rosanova et al., 2009). Secondly, it is possible that the different frequency responses in each cortical area might reflect different anatomical background.

Indeed, recent studies have reported that there is a strong correlation between cytoarchitecture and anatomical and functional connectivity in cat, macaque and humans (Beul et al., 2015; Scholtens et al., 2014), with superior parietal showing both a different cytoarchitecture as well as a different connectivity architecture than supplementary motor regions (van den Heuvel et al., 2015). This might explain why the functional activation at specific resonant frequencies is related to the structural coupling (i.e., the amount of tracts connecting them) differently depending on the anatomical architecture of the specific brain region.

\section{Limitations}

Given the intrinsic limitations of the EEG in terms of spatial resolution, it is important to stress that the patterns of connectivity detected by TMS/hd-EEG are necessarily coarse. Even though TEPs are characterized by a good test-retest reproducibility (Lioumis et al., 2009), the interindividual reproducibility of the outgoing flow of information could be improved by a better 
computation of the electric field induced by TMS. More advanced models (boundary, or finite, element models) could improve the accuracy of the source localization (Wagner et al., 2009). Another limitation of our study concerns the relatively small sample size and the inter-subject variability both at the TMS/hd-EEG response and the tractography level. However, in this paper we investigated for the first time through TMS-EEG and DWI the relationship between structural and directed functional connectivity patterns in healthy subjects. The number of subjects included in the study, coupled with appropriate statistics, can definitely be of use for future research and hypothesis. Furthermore, the functional and structural connectivity profiles obtained from our cohort appear to be stable across subjects (see also Supplementary Figure S4).

In addition, it has been shown that there are many brain regions with complex structural architecture, also referred to as "crossing fibers" (Jeurissen et al., 2011; Tournier et al., 2012). In this context, tractography approaches based on more advanced diffusion models (Jeurissen et al., 2011), or on more refined anatomical constraints (Smith et al., 2012) may provide more accurate anatomical connectivity patterns of brain networks. Therefore, our approach works best for studying large scale interactions than fine scale, local dynamics.

Finally, a b-value of $1000 \mathrm{~s} / \mathrm{mm}^{2}$ is lower than the optimal one for performing CSD, about 2500-3000 s/mm (Tournier et al., 2013). However, despite of a low b-value, with a sufficient amount of directions crossing fibers can be reliably modeled with CSD and the result is still significantly better than with a simple DTI-based model, e.g., see (Roine et al., 2015) for a successful application.

\section{Conclusions}

In conclusion, the findings of the study suggest that the way brain structure and function interact 26 
after TMS perturbation follows a rather complex and multifaceted dynamic. Notably, when looking at the whole-brain network level, the structure/function relationship is largely reduced following TMS within each frequency band (Fig. 5). Nonetheless, when looking at the local responses of the target regions, we showed that the way directed functional connectivity changes due to TMS might depend on both the frequency at which the cortical module is elicited by TMS, and on the structural architecture of the specific stimulated cortical region (Fig. 6). Future studies should validate this hypothesis by exploring natural frequency profiles in different sites of stimulation and/or by evaluating structure/function correlations at natural peak frequencies in brain regions other than the stimulated ones. An interesting follow-up of this study would be to investigate how the TMS pulse changes the functional connectivity profile of different stimulated sites over shorter time periods, i.e., by using shorter time windows or a sliding window approach. In conclusion, our multimodal whole-brain approach gives new insight on how TMS interferes with the brain network in healthy controls. More specifically, our study points out the importance of taking into account the major role played by different cortical oscillations when investigating the mechanisms for integration and segregation of information in the human brain (Casali et al., 2013). Another interesting follow-up of this study would indeed be to look at differences in structure-function interactions either when the cognitive function is pharmacologically modulated (i.e., anesthesia), or following pathology, damage or disruption in structural connections (i.e., coma and disorders of consciousness).

\section{Acknowledgements}

We thank Timo Roine, Erik Ziegler, Gianluca Frasso, Andrea Piarulli and Georgos Antonopoulos for the insightful discussion and comments on the manuscript. We thank Marie-Aurelie Bruno, Athena Demertzi, Audrey Vanhaudenhuyse and Melanie Boly for help in 
acquiring the data. This research was supported by the Wallonia-Brussels Federation of Concerted Research Action (ARC), the European Union's Horizon 2020 Research and Innovation Program under Grant Agreement No. 720270 (HBP SGA1), LUMINOUS project (EU-H2020-FETOPEN-GA686764), Fonds National de la Recherche Scientifique de Belgique (FNRS), Belgian Science Policy (CEREBNET, BELSPO), McDonnell Foundation, European Space Agency, Mind Science Foundation, University Hospital and University of Liège. OB is a research fellow, OG a post-doctoral fellow and SL a research director at FNRS.

\section{Disclosure Statement}

No competing financial interests exist.

\section{References}

Arnold, M., Milner, X.H.R., Witte, H., Bauer, R., Braun, C., 1998. Adaptive AR modeling of nonstationary time series by means of Kalman filtering. Biomedical Engineering, IEEE Transactions on 45, 553-562.

Astolfi, L., Cincotti, F., Mattia, D., de Vico Fallani, F., Tocci, A., Colosimo, A., Salinari, S., Marciani, M.G., Hesse, W., Witte, H., others, 2008. Tracking the time-varying cortical connectivity patterns by adaptive multivariate estimators. Biomedical Engineering, IEEE Transactions on 55, 902-913.

Barttfeld, P., Uhrig, L., Sitt, J.D., Sigman, M., Jarraya, B.e., chir, Dehaene, S., 2015. Signature of consciousness in the dynamics of resting-state brain activity. Proceedings of the National Academy of Sciences 112, 887-892.

Berg, P., Scherg, M., 1994. A fast method for forward computation of multiple-shell spherical head models. Electroencephalography and clinical neurophysiology 90, 58-64.

Beul, S.F., Grant, S., Hilgetag, C.C., 2015. A predictive model of the cat cortical connectome based on cytoarchitecture and distance. Brain Structure and Function 220, 3167-3184.

Bonato, C., Miniussi, C., Rossini, P.M., 2006. Transcranial magnetic stimulation and cortical evoked potentials: a TMS/EEG co-registration study. Clinical neurophysiology 117, 1699-1707.

Bortoletto, M., Veniero, D., Thut, G., Miniussi, C., 2015. The contribution of TMS--EEG coregistration 
in the exploration of the human cortical connectome. Neuroscience \\& Biobehavioral Reviews 49, 114-124.

Bullmore, E., Sporns, O., 2009. Complex brain networks: graph theoretical analysis of structural and functional systems. Nat Rev Neurosci 10, 186-198.

Caeyenberghs, K., Leemans, A., De Decker, C., Heitger, M., Drijkoningen, D., Linden, C.V., Sunaert, S., Swinnen, S.P., 2012. Brain connectivity and postural control in young traumatic brain injury patients: A diffusion MRI based network analysis. Neurolmage: clinical 1, 106-115.

Casali, A.G., Casarotto, S., Rosanova, M., Mariotti, M., Massimini, M., 2010. General indices to characterize the electrical response of the cerebral cortex to TMS. Neuroimage 49, 1459-1468.

Casali, A.G., Gosseries, O., Rosanova, M., Boly, M.e., lanie, Sarasso, S., Casali, K.R., Casarotto, S., Bruno, M.-A.e., lie, Laureys, S., Tononi, G., others, 2013. A theoretically based index of consciousness independent of sensory processing and behavior. Science translational medicine 5, 198ra105-198ra105.

Casarotto, S., Lauro, L.J.R., Bellina, V., Casali, A.G., Rosanova, M., Pigorini, A., Defendi, S., Mariotti, M., Massimini, M., 2010. EEG responses to TMS are sensitive to changes in the perturbation parameters and repeatable over time. PLoS One 5, e10281.

Catani, M., Howard, R.J., Pajevic, S., Jones, D.K., 2002. Virtual in Vivo Interactive Dissection of White Matter Fasciculi in the Human Brain. Neuroimage 17, 77-94.

Chu, C.J., Tanaka, N., Diaz, J., Edlow, B.L., Wu, O., H\"a, m.a., I\"a,inen, M., Stufflebeam, S., Cash, S.S., Kramer, M.A., 2015. EEG functional connectivity is partially predicted by underlying white matter connectivity. Neuroimage 108, 23-33.

Cona, F., Zavaglia, M., Massimini, M., Rosanova, M., Ursino, M., 2011. A neural mass model of interconnected regions simulates rhythm propagation observed via TMS-EEG. Neurolmage 57, 1045-1058.

De Geeter, N., Crevecoeur, G., Leemans, A., others, 2015. Effective electric fields along realistic DTI-based neural trajectories for modelling the stimulation mechanisms of TMS. Physics in medicine and biology 60, 453.

Efron, B., Tibshirani, R., 1986. Bootstrap methods for standard errors, confidence intervals, and other measures of statistical accuracy. Statistical science, 54-75.

Ferrarelli, F., Sarasso, S., Guller, Y., Riedner, B.A., Peterson, M.J., Bellesi, M., Massimini, M., Postle, B.R., Tononi, G., 2012. Reduced natural oscillatory frequency of frontal thalamocortical circuits in schizophrenia. Archives of general psychiatry 69, 766-774.

Gosseries, O., Sarasso, S., Casarotto, S., Boly, M.e., lanie, Schnakers, C., Napolitani, M., Bruno, M.-A.e., lie, Ledoux, D., Tshibanda, J.-F., Massimini, M., others, 2015. On the Cerebral Origin of EEG Responses to TMS: Insights From Severe Cortical Lesions. Brain stimulation 8, 142-149.

Granger, C.W., 1969. Investigating causal relations by econometric models and cross-spectral methods. Econometrica: Journal of the Econometric Society, 424-438.

Groppa, S., Muthuraman, M., Otto, B., Deuschl, G.u., nther, Siebner, H.R., Raethjen, J., 2013. Subcortical substrates of TMS induced modulation of the cortico-cortical connectivity. Brain stimulation 6, 138-146.

Hagmann, P., Cammoun, L., Gigandet, X., Meuli, R., Honey, C.J., Wedeen, V.J., Sporns, O., 2008. Mapping the structural core of human cerebral cortex. PLoS biology 6, e159.

IImoniemi, R.J., Virtanen, J., Ruohonen, J., Karhu, J., Aronen, H.J., Katila, T., others, 1997. Neuronal responses to magnetic stimulation reveal cortical reactivity and connectivity. Neuroreport 8 , 
3537-3540.

Jeurissen, B., Leemans, A., Jones, D.K., Tournier, J.-D., Sijbers, J., 2011. Probabilistic fiber tracking using the residual bootstrap with constrained spherical deconvolution. Human brain mapping 32, 461-479.

Jones, D.K., Horsfield, M.A., Simmons, A., 1999. Optimal strategies for measuring diffusion in anisotropic systems by magnetic resonance imaging. Magn Reson Med 42.

Kaminski, M.J., Blinowska, K.J., 1991. A new method of the description of the information flow in the brain structures. Biological cybernetics 65, 203-210.

Leemans, A., Jones, D.K., 2009. The B-matrix must be rotated when correcting for subject motion in DTI data. Magnetic Resonance in Medicine 61, 1336-1349.

Lioumis, P., Ki\vc, i\'c,, Dubravko, Savolainen, P., M\"a, k.a., , Jyrki P., K\"a, h.o., nen, Seppo, 2009. Reproducibility of TMS-Evoked EEG responses. Human brain mapping 30, 1387-1396.

Liu, Q., Balsters, J.H., Baechinger, M., van der Groen, O., Wenderoth, N., Mantini, D., 2015. Estimating a neutral reference for electroencephalographic recordings: the importance of using a high-density montage and a realistic head model. J Neural Eng 12, 056012.

Massimini, M., Ferrarelli, F., Huber, R., Esser, S.K., Singh, H., Tononi, G., 2005. Breakdown of cortical effective connectivity during sleep. Science 309, 2228-2232.

Mattout, J.e., r'e,mie, Phillips, C., Penny, W.D., Rugg, M.D., Friston, K.J., 2006. MEG source localization under multiple constraints: an extended Bayesian framework. Neurolmage 30, 753-767.

Phillips, C., Mattout, J., Rugg, M.D., Maquet, P., Friston, K.J., 2005. An empirical Bayesian solution to the source reconstruction problem in EEG. Neurolmage 24, 997-1011.

Rogasch, N.C., Fitzgerald, P.B., 2013. Assessing cortical network properties using TMS--EEG. Human brain mapping 34, 1652-1669.

Rogasch, N.C., Thomson, R.H., Daskalakis, Z.J., Fitzgerald, P.B., 2013. Short-Latency Artifacts Associated with Concurrent TMS--EEG. Brain stimulation 6, 868-876.

Roine, U., Salmi, J., Roine, T., Nieminen-von Wendt, T., Lepp\"a, m.a., ki, Sami, Rintahaka, P., Tani, P., Leemans, A., Sams, M., 2015. Constrained spherical deconvolution-based tractography and tract-based spatial statistics show abnormal microstructural organization in Asperger syndrome. Molecular autism 6, 1-12.

Rosanova, M., Casali, A., Bellina, V., Resta, F., Mariotti, M., Massimini, M., 2009. Natural frequencies of human corticothalamic circuits. The Journal of Neuroscience 29, 7679-7685.

Rosanova, M., Gosseries, O., Casarotto, S., Boly, M.e., lanie, Casali, A.G., Bruno, M.-A.e., lie, Mariotti, M., Boveroux, P., Tononi, G., Laureys, S., others, 2012. Recovery of cortical effective connectivity and recovery of consciousness in vegetative patients. Brain, awr340.

Rubinov, M., Sporns, O., 2010. Complex network measures of brain connectivity: uses and interpretations. Neuroimage 52, 1059-1069.

Scholtens, L.H., Schmidt, R., de Reus, M.A., van den Heuvel, M.P., 2014. Linking macroscale graph analytical organization to microscale neuroarchitectonics in the macaque connectome. The Journal of Neuroscience 34, 12192-12205.

Smith, R.E., Tournier, J.-D., Calamante, F., Connelly, A., 2012. Anatomically-constrained tractography: improved diffusion MRI streamlines tractography through effective use of anatomical information. Neuroimage 62, 1924-1938.

Smith, S.M., Jenkinson, M., Woolrich, M.W., Beckmann, C.F., Behrens, T.E.J., Johansen-Berg, H., Bannister, P.R., De Luca, M., Drobnjak, I., Flitney, D.E., others, 2004. Advances in functional and 
structural MR image analysis and implementation as FSL. Neuroimage 23, S208-S219.

Song, S.-K., Sun, S.-W., Ramsbottom, M.J., Chang, C., Russell, J., Cross, A.H., 2002. Dysmyelination revealed through MRI as increased radial (but unchanged axial) diffusion of water. Neuroimage 17, 1429-1436.

Sporns, O., 2011. Networks of the Brain. MIT press.

Sporns, O., Tononi, G., K\"o, t., Rolf, 2005. The human connectome: a structural description of the human brain. PLoS computational biology 1 , e42.

Tournier, J., Calamante, F., Connelly, A., others, 2012. MRtrix: diffusion tractography in crossing fiber regions. International Journal of Imaging Systems and Technology 22, 53-66.

Tournier, J., Calamante, F., Connelly, A., others, 2013. Determination of the appropriate b value and number of gradient directions for high-angular-resolution diffusion-weighted imaging. NMR in Biomedicine 26, 1775-1786.

Tournier, J., Calamante, F., Gadian, D.G., Connelly, A., others, 2004. Direct estimation of the fiber orientation density function from diffusion-weighted MRI data using spherical deconvolution. Neurolmage 23, 1176-1185.

Tournier, J., Yeh, C.-H., Calamante, F., Cho, K.-H., Connelly, A., Lin, C.-P., others, 2008. Resolving crossing fibres using constrained spherical deconvolution: validation using diffusion-weighted imaging phantom data. Neuroimage 42, 617-625.

Tzourio-Mazoyer, N., Landeau, B., Papathanassiou, D., Crivello, F., Etard, O., Delcroix, N., Mazoyer, B., Joliot, M., 2002. Automated anatomical labeling of activations in SPM using a macroscopic anatomical parcellation of the MNI MRI single-subject brain. Neuroimage 15, 273-289.

van den Heuvel, M.P., Scholtens, L.H., Barrett, L.F., Hilgetag, C.C., de Reus, M.A., 2015. Bridging cytoarchitectonics and connectomics in human cerebral cortex. The Journal of Neuroscience 35, 13943-13948.

Van Der Werf, Y.D., Paus, T.a., \Vs,,, 2006. The neural response to transcranial magnetic stimulation of the human motor cortex. I. Intracortical and cortico-cortical contributions. Experimental brain research 175, 231-245.

Van Mierlo, P., Carrette, E., Hallez, H., Raedt, R., Meurs, A., Vandenberghe, S., Roost, D., Boon, P., Staelens, S., Vonck, K., 2013. Ictal-onset localization through connectivity analysis of intracranial EEG signals in patients with refractory epilepsy. Epilepsia 54, 1409-1418.

Van Mierlo, P., Carrette, E., Hallez, H., Vonck, K., Van Roost, D., Boon, P., Staelens, S., 2011. Accurate epileptogenic focus localization through time-variant functional connectivity analysis of intracranial electroencephalographic signals. Neuroimage 56, 1122-1133.

Voineskos, A.N., Farzan, F., Barr, M.S., Lobaugh, N.J., Mulsant, B.H., Chen, R., Fitzgerald, P.B., Daskalakis, Z.J., 2010. The role of the corpus callosum in transcranial magnetic stimulation induced interhemispheric signal propagation. Biological psychiatry 68, 825-831.

Wagner, T., Rushmore, J., Eden, U., Valero-Cabre, A., 2009. Biophysical foundations underlying TMS: setting the stage for an effective use of neurostimulation in the cognitive neurosciences. Cortex 45 , 1025-1034.

Wilke, C., Ding, L., He, B., 2008. Estimation of time-varying connectivity patterns through the use of an adaptive directed transfer function. Biomedical Engineering, IEEE Transactions on 55, 2557-2564.

Xia, M., Wang, J., He, Y., others, 2013. BrainNet Viewer: a network visualization tool for human brain connectomics. PloS one 8, e68910.

Yao, D., 2001. A method to standardize a reference of scalp EEG recordings to a point at infinity. 
Physiol Meas 22, 693-711.

Zalesky, A., Akhlaghi, H., Corben, L.A., Bradshaw, J.L., Delatycki, M.B., Storey, E., Georgiou-Karistianis, N., Egan, G.F., 2014. Cerebello-cerebral connectivity deficits in Friedreich ataxia. Brain Structure and Function 219, 969-981. 